18-20 June, 2021

\title{
The Mediating Role of Interpersonal Emotion Regulation Strategies and General Belongingness in Relation to Childhood Emotional Abuse and Meaning in Life
}

\author{
Letisya Yancı Kumaşoğlu \\ Başak Türküler Aka
}

The Graduate School of Social Sciences, Bahçeşehir University, Turkey

\begin{abstract}
Childhood is a period that care and love are needed most for both physical and mental health. Many psychological theories focus on childhood life experiences since it is a significant indicator of psychological well-being. Especially, childhood emotional abuse is a phenomenon that has long-lasting consequences on human life. The purpose of the study was to examine the relationship between childhood emotional abuse and meaning in life, interpersonal emotion regulation strategies as enhancing positive effect, perspective taking, soothing and social modeling, and general belongingness as mediators. To examine this relationship, the Socio-Demographic Information Form, Childhood Trauma Questionnaire, Interpersonal Emotion Regulation Questionnaire, Meaning in Life Questionnaire and General Belongingness Scale was used. This study was conducted with 307 participants ages between 18 and $65(M=31.38 ; S D=11.06)$. Results of the study showed that enhancing positive affect and general belongingness fully mediated the relationship between childhood emotional abuse and the search for meaning in life. Moreover, perspective-taking, and general belongingness fully mediated the relationship between childhood emotional abuse and the presence of meaning in life. The findings were discussed in line with the current literature.
\end{abstract}

Keywords: Belongingness; Childhood Trauma; Emotional Abuse; Emotion Regulation;

Meaning in Life 